\title{
Remarks on the operator-norm convergence of the Trotter product formula
}

\author{
Hagen NeIdhardT, Artur Stephan , and Valentin A. ZaGrebnOV $^{\ddagger}$
}

March 29, 2017

\begin{abstract}
We revise the operator-norm convergence of the Trotter product formula for a pair $\{A, B\}$ of generators of semigroups on a Banach space. Operator-norm convergence holds true if the dominating operator $A$ generates a holomorphic contraction semigroup and $B$ is a $A$-infinitesimally small generator of a contraction semigroup, in particular, if $B$ is a bounded operator. Inspired by studies of evolution semigroups it is shown in the present paper that the operator-norm convergence generally fails even for bounded operators $B$ if $A$ is not a holomorphic generator. Moreover, it is shown that operator norm convergence of the Trotter product formula can be arbitrary slow.
\end{abstract}

Keywords: Semigroups, bounded perturbations, Trotter product formula, Darboux-Riemann sums, operator-norm convergence.

\section{Introduction and main results}

Recall that the product formula

$$
e^{-\tau C}=\lim _{n \rightarrow \infty}\left(e^{-\tau A / n} e^{-\tau B / n}\right)^{n}, \quad \tau \geq 0,
$$

was established by S. Lie (in 1875) for matrices where $C:=A+B$. The proof is based on the telescopic representation

$$
\left(e^{-\tau A / n} e^{-\tau B / n}\right)^{n}-e^{-\tau C}=\sum_{k=0}^{n-1}\left(e^{-\tau A / n} e^{-\tau B / n}\right)^{n-1-k}\left(e^{-\tau A / n} e^{-\tau B / n}-e^{-\tau C / n}\right) e^{-k \tau C / n},
$$

$n \in \mathbb{N}$, and expansion

$$
e^{-\tau X}=I-\tau X+O\left(\tau^{2}\right), \quad \tau \longrightarrow 0,
$$

for a matrix $X$ in the operator-norm topology $\|\cdot\|$. Indeed, using this expansion one obtains the estimate:

$$
\left\|e^{-\tau A / n} e^{-\tau B / n}-e^{-\tau C / n}\right\|=O\left((\tau / n)^{2}\right) .
$$

\footnotetext{
*H. Neidhardt: WIAS Berlin, Mohrenstr. 39, D-10117 Berlin, Germany; email: hagen.neidhardt@wias-berlin.de

${ }^{\dagger}$ A. Stephan: HU Berlin, Institut für Mathematik, Unter den Linden 6, D-10099 Berlin, Germany; email: stephan@math.hu-berlin.de

${ }^{\ddagger}$ V.A.Zagrebnov: Université d’Aix-Marseille - Institut de Mathématiques de Marseille (UMR 7373), CMI - Technopôle Château-Gombert, 39, rue F. Joliot Curie, 13453 Marseille, France, email: valentin.zagrebnov@univ-amu.fr
} 
Then from (1.1) we get the existence of a constant $c_{0}>0$ such that the following estimate holds

$$
\left\|\left(e^{-\tau A / n} e^{-\tau B / n}\right)^{n}-e^{-\tau C}\right\| \leq c_{0} \frac{\tau^{2}}{n^{2}} \sum_{k=0}^{n-1} e^{\tau \frac{n-1-k}{n}} \tau\|A\| e^{\tau \frac{n-1-k}{n}} \tau\|B\| e^{\tau \frac{k}{n}\|C\|} .
$$

Since $\|C\| \leq\|A\|+\|B\|$, one obtains inequality

$$
\left\|\left(e^{-\tau A / n} e^{-\tau B / n}\right)^{n}-e^{-\tau C}\right\| \leq c_{0} \frac{\tau^{2}}{n^{2}} \sum_{k=0}^{n-1} e^{\tau \frac{n-1}{n}(\|A\|+\|B\|)} \leq c_{0} \frac{\tau^{2}}{n} e^{\tau(\|A\|+\|B\|)},
$$

which yields that

$$
\sup _{\tau \in[0, T]}\left\|\left(e^{-\tau A / n} e^{-\tau B / n}\right)^{n}-e^{-\tau C}\right\|=O(1 / n),
$$

as $n \rightarrow \infty$ for any $T>0$. Note that this proof carries through verbatim for bounded operators $A$ and $B$ on Banach spaces.

H. Trotter [7] has extended this result to unbounded operators $A$ and $B$ on Banach spaces, but in the strong operator topology. He proved that if $A$ and $B$ are generators of contractions semigroups on a separable Banach space such that the algebraic sum $A+B$ is a densely defined closable operator and the closure $C=\overline{A+B}$ is a generator of a contraction semigroup, then

$$
e^{-\tau C}=\mathrm{s}-\lim _{n \rightarrow \infty}\left(e^{-\tau A / n} e^{-\tau B / n}\right)^{n},
$$

uniformly in $\tau \in[0, T]$ for any $T>0$. It is obvious that this result holds if $B$ is a bounded operator.

Considering the Trotter product formula on a Hilbert space T. Kato has shown in 4 that for nonnegative operators $A$ and $B$ the Trotter formula (1.3) holds in the strong operator topology if $\operatorname{dom}(\sqrt{A}) \cap$ $\operatorname{dom}(\sqrt{B})$ is dense in the Hilbert space and $C=A \dot{+} B$ is the form-sum of operators $A$ and $B$. Later on it was shown in [3] that the relation (1.2) holds if the algebraic sum $C=A+B$ is already a self-adjoint operator. Therefore, (1.2) is valid in particular, if $B$ is a bounded self-adjoint operator.

The historically first result concerning the operator-norm convergence of the Trotter formula in a Banach space is due to [1. Since the concept of self-adjointness is missing for Banach spaces it was assumed that the dominating operator $A$ is a generator of a contraction holomorphic semigroup and $B$ is a generator of a contraction semigroup. In Theorem 3.6 of [1] it was shown that if $0 \in \rho(A)$ and if there is a $\alpha \in[0,1) \operatorname{such}$ that $\operatorname{dom}\left(A^{\alpha}\right) \subseteq \operatorname{dom}(B)$ and $\operatorname{dom}\left(A^{*}\right) \subseteq \operatorname{dom}\left(B^{*}\right)$, then for any $T>0$ one has

$$
\sup _{\tau \in[0, T]}\left\|\left(e^{-\tau A / n} e^{-\tau B / n}\right)^{n}-e^{-\tau C}\right\|=O\left(\ln (n) / n^{1-\alpha}\right) .
$$

Note that the assumption $0 \in \rho(A)$ was made for simplicity and that the assumption $\operatorname{dom}\left(A^{\alpha}\right) \subseteq$ $\operatorname{dom}(B)$ yields that the operator $B$ is infinitesimally small with respect to $A$. Taking into account $[5$, Corollary IX.2.5] one gets that the well-defined algebraic sum $C=A+B$ is a generator of a contraction holomorphic semigroup. By Theorem 3.6 of [1] the convergence rate (1.4) improves if $B$ is a bounded operator, i.e. $\alpha=0$. Then for any $T>0$ one gets

$$
\sup _{\tau \in[0, T]}\left\|\left(e^{-\tau A / n} e^{-\tau B / n}\right)^{n}-e^{-\tau C}\right\|=O\left((\ln (n))^{2} / n\right) .
$$

Summarizing, the question arises whether the Trotter product formula converges in the operator-norm if $A$ is a generator of a contraction (but not holomorphic) semigroup and $B$ is a bounded operator? The aim of the present paper is to give an answer to this question for a certain class of generators. 
It turns out that an appropriate class for that is the class of generators of evolution semigroups. To proceed further we need the notion of a propagator, or a solution operator [6].

A strongly continuous map $U(\cdot, \cdot): \Delta \longrightarrow \mathcal{B}(X)$, where $\Delta:=\{(t, s): 0<s \leq t \leq T\}$ and $\mathcal{B}(X)$ is the set of bounded operators on the separable Banach space $X$, is called a propagator if the conditions

$$
\begin{aligned}
& \text { (i) } \sup _{(t, s) \in \Delta}\|U(t, s)\|_{\mathcal{B}(X)}<\infty, \\
& \text { (ii) } U(t, s)=U(t, r) U(r, s), 0<s \leq r \leq t \leq T,
\end{aligned}
$$

are satisfied. Let us consider the Banach space $L^{p}(\mathcal{I}, X), \mathcal{I}:=[0, T], p \in[1, \infty)$. The operator $\mathcal{K}$ is an evolution generator of the evolution semigroup $\left\{e^{-\tau \mathcal{K}}\right\}_{\tau \geq 0}$ if there is a propagator such that the representation

$$
\left(e^{-\tau \mathcal{K}} f\right)(t)=U(t, t-\tau) \chi_{\mathcal{I}}(t-\tau) f(t-\tau), \quad f \in L^{p}(\mathcal{I}, X)
$$

holds for a.e. $t \in \mathcal{I}$ and $\tau \geq 0$ [6]. Since $e^{-\tau \mathcal{K}} f=0$ for $\tau \geq T$, the evolution generator $\mathcal{K}$ can never be a generator of a holomorphic semigroup.

A simple example of an evolution generator is the differentiation operator:

$$
\begin{aligned}
\left(D_{0} f\right)(t) & :=\partial_{t} f(t), \\
f \in \operatorname{dom}\left(D_{0}\right) & :=\left\{f \in H^{1, p}(\mathcal{I}, X): f(0)=0\right\} .
\end{aligned}
$$

Then by (1.6) one obviously gets the contraction shift semigroup:

$$
\left(e^{-\tau D_{0}} f\right)(t)=\chi_{\mathcal{I}}(t-\tau) f(t-\tau), \quad f \in L^{p}(\mathcal{I}, X),
$$

for a.e. $t \in \mathcal{I}$ and $\tau \geq 0$. Hence, (1.5) implies that the corresponding propagator of the non-holomorphic evolution semigroup $\left\{e^{-\tau D_{0}}\right\}_{\tau \geq 0}$ is given by $U_{D_{0}}(t, s)=I,(t, s) \in \Delta$.

Note that in [6] we considered the operator $\mathcal{K}_{0}:=\overline{D_{0}+\mathcal{A}}$, where $\mathcal{A}$ is the multiplication operator induced by a generator $A$ of a holomorphic contraction semigroup on $X$. More precisely

$$
\begin{aligned}
(\mathcal{A} f)(t) & :=A f(t), \text { and }\left(e^{-\tau \mathcal{A}} f\right)(t)=e^{-\tau A} f(t), \\
f \in \operatorname{dom}(\mathcal{A}) & :=\left\{f \in L^{p}(\mathcal{I}, X): A f(\cdot) \in L^{p}(\mathcal{I}, X)\right\} .
\end{aligned}
$$

Then the perturbation of the shift semigroup (1.7) by $\mathcal{A}$ corresponds to the semigroup with generator $\mathcal{K}_{0}$. One easily checks that $\mathcal{K}_{0}$ is an evolution generator of a contraction semigroup on $L^{p}(\mathcal{I}, X)$ that is never holomorphic. Indeed, since the generators $D_{0}$ and $\mathcal{A}$ commute, the representation (1.5) for evolution semigroup $\left\{e^{-\tau \mathcal{K}_{0}}\right\}_{\tau \geq 0}$ takes the form:

$$
\left(e^{-\tau \mathcal{K}_{0}} f\right)(t)=e^{-\tau A} \chi_{\mathcal{I}}(t-\tau) f(t-\tau), \quad f \in L^{p}(\mathcal{I}, X),
$$

for a.e. $t \in \mathcal{I}$ and $\tau \geq 0$ with propagator $U_{0}(t, s)=e^{-(t-s) A}$. Therefore, again $e^{-\tau \mathcal{K}_{0}} f=0$ for $\tau \geq T$.

Furthermore, if $B(\cdot)$ is a strongly measurable family of generators of contraction semigroups on $X$, i.e. $B(\cdot): \mathcal{I} \longrightarrow \mathcal{G}(1,0)$ (see [4], Ch.IX, $\S 1.4$ ), then the induced multiplication operator $\mathcal{B}:$

$$
\begin{aligned}
(\mathcal{B} f)(t) & :=B(t) f(t), \\
f \in \operatorname{dom}(\mathcal{B}) & :=\left\{f \in L^{p}(\mathcal{I}, X): \begin{array}{c}
f(t) \in \operatorname{dom}(B(t)) \text { for a.e. } t \in \mathcal{I} \\
B(t) f(t) \in L^{p}(\mathcal{I}, X)
\end{array}\right\},
\end{aligned}
$$

is a generator of a contraction semigroup on $L^{p}(\mathcal{I}, X)$.

In [6] it was assumed that $\{B(t)\}_{t \in \mathcal{I}}$ is a strongly measurable family of generators of contraction semigroups and that $A$ is a generator of a bounded holomorphic semigroup with $0 \in \rho(A)$ for simplicity. Moreover, we supposed that the following conditions are satisfied: 
(i) $\operatorname{dom}\left(A^{\alpha}\right) \subseteq \operatorname{dom}(B(t))$ for a.e. $t \in \mathcal{I}$ and some $\alpha \in(0,1)$ such that

$$
\underset{t \in \mathcal{I}}{\operatorname{ess} \sup }\left\|B(t) A^{-\alpha}\right\|_{\mathcal{B}(X)}<\infty ;
$$

(ii) $\operatorname{dom}\left(A^{*}\right) \subseteq \operatorname{dom}\left(B(t)^{*}\right)$ for a.e. $t \in \mathcal{I}$ such that

$$
\underset{t \in \mathcal{I}}{\operatorname{ess} \sup }\left\|B(t)^{*}\left(A^{-1}\right)^{*}\right\|_{\mathcal{B}(X)}<\infty
$$

(iii) there is a $\beta \in(\alpha, 1)$ and $L_{\beta}>0$ such that

$$
\left\|A^{-1}(B(t)-B(s)) A^{-\alpha}\right\|_{\mathcal{B}(X)} \leq L_{\beta}|t-s|^{\beta}, \quad t, s \in \mathcal{I} .
$$

Under these assumptions it turns out that $\mathcal{K}:=\mathcal{K}_{0}+\mathcal{B}$ is a generator of a contraction evolution semigroup, i.e there is a propagator $\{U(t, s)\}_{(t, s) \in \Delta}$ such that the representation (1.5) is valid. Moreover, we prove in [6] the Trotter product formula converges in the operator norm with convergence rate $O\left(1 / n^{\beta-\alpha}\right)$ :

$$
\sup _{\tau \geq 0}\left\|\left(e^{-\tau \mathcal{K}_{0} / n} e^{-\tau \mathcal{B} / n}\right)^{n}-e^{-\tau \mathcal{K}}\right\|_{\mathcal{B}\left(L^{p}(\mathcal{I}, X)\right)}=O\left(1 / n^{\beta-\alpha}\right) .
$$

We comment that if $B(\cdot): \mathcal{I} \longrightarrow \mathcal{B}(X)$ is a Hölder continuous function with Hölder exponent $\beta \in(0,1)$, then the assumptions (i)-(iii) are satisfied for any $\alpha \in(0, \beta)$. Then our results [6] yield that

$$
\sup _{\tau \geq 0}\left\|\left(e^{-\tau \mathcal{K}_{0} / n} e^{-\tau \mathcal{B} / n}\right)^{n}-e^{-\tau \mathcal{K}}\right\|_{\mathcal{B}\left(L^{p}(\mathcal{I}, X)\right)}=O\left(1 / n^{\gamma}\right)
$$

holds for any $\gamma \in(0, \beta)$. Moreover, in this case the perturbation of the shift semigroup (1.7) by a bounded generator (1.8) gives an evolution semigroup with generator $D_{0}+\mathcal{B}$. Then as a corollary of (1.10) for $\mathcal{A}=0$, we get the Trotter product estimate

$$
\sup _{\tau \geq 0}\left\|\left(e^{-\tau D_{0} / n} e^{-\tau \mathcal{B} / n}\right)^{n}-e^{-\tau\left(D_{0}+\mathcal{B}\right)}\right\|_{\mathcal{B}\left(L^{p}(\mathcal{I}, X)\right)}=O\left(1 / n^{\gamma}\right) .
$$

The aim of our note is to show that the convergence rate (1.11) is close to the optimal one. To this end we consider the simple case, when $X=\mathbb{C}$ and we put for simplicity $\mathcal{I}:=[0,1]$.

The main results of this paper can be summarized as follows:

If the operator $\mathcal{B}$ is equal to the multiplication operator $Q$ induced by a bounded measurable function $q(\cdot): \mathcal{I} \longrightarrow \mathbb{C}$ in $L^{p}(\mathcal{I})$, then one can verify that the condition $(1.9)$ is equivalent to $q(\cdot) \in C^{0, \beta}(\mathcal{I})$, see definition below. In this case the convergence rate is

$$
\sup _{\tau \geq 0}\left\|e^{-\tau\left(D_{0}+Q\right)}-\left(e^{-\tau D_{0} / n} e^{-\tau Q / n}\right)^{n}\right\|_{\mathcal{B}\left(L^{p}(\mathcal{I}, X)\right)}=O\left(1 / n^{\beta}\right) .
$$

This result remains true if $q(\cdot)$ is Lipschitz continuous, i.e. $\beta=1$. But if $q(\cdot)$ is only continuous, then

$$
\sup _{\tau \geq 0}\left\|e^{-\tau\left(D_{0}+Q\right)}-\left(e^{-\tau D_{0} / n} e^{-\tau Q / n}\right)^{n}\right\|_{\mathcal{B}\left(L^{p}(\mathcal{I}, X)\right)}=o(1) .
$$

Moreover, for any convergent to zero sequence $\delta_{n}>0, n \in \mathbb{N}$, there exists a continuous function $q(\cdot)$ such that

$$
\sup _{\tau \geq 0}\left\|e^{-\tau\left(D_{0}+Q\right)}-\left(e^{-\tau D_{0} / n} e^{-\tau Q / n}\right)^{n}\right\|_{\mathcal{B}\left(L^{p}(\mathcal{I}, X)\right)}=\omega\left(\delta_{n}\right)
$$


where the Landau symbol $\omega(\cdot)$ is defined below.

Finally, there is an example of a bounded measurable function $q(\cdot)$ such that

$$
\limsup _{n \rightarrow \infty} \sup _{\tau \geq 0}\left\|e^{-\tau\left(D_{0}+Q\right)}-\left(e^{-\tau D_{0} / n} e^{-\tau Q / n}\right)^{n}\right\|_{\mathcal{B}\left(L^{p}(\mathcal{I}, X)\right)}>0 .
$$

Hence, in contrast to the holomorphic case, when the dominating operator is a generator of a holomorphic semigroup (1.4), the Trotter product formula (1.15) with dominating generator $D_{0}$, may not converge in the operator-norm.

The paper is organized as follows. In Section 2 we reformulate the convergence of the Trotter product formula in terms of the corresponding evolutions semigroups. In Section 3 we prove the results (1.12)(1.15).

We conclude this section by few remarks concerning notation used in this paper.

1. We use a definition of the generator $C$ of a semigroup (1.3), which differs from the standard one by a minus [5].

2. Furthermore, we widely use the so-called Landau symbols:

$$
\begin{aligned}
& g(n)=O(f(n)) \Longleftrightarrow \limsup _{n \rightarrow \infty}\left|\frac{g(n)}{f(n)}\right|<\infty, \\
& g(n)=o(f(n)) \Longleftrightarrow \limsup _{n \rightarrow \infty}\left|\frac{g(n)}{f(n)}\right|=0, \\
& g(n)=\Theta(f(n)) \Longleftrightarrow 0<\liminf _{n \rightarrow \infty}\left|\frac{g(n)}{f(n)}\right| \leq \limsup _{n \rightarrow \infty}\left|\frac{g(n)}{f(n)}\right|<\infty, \\
& g(n)=\omega(f(n)) \Longleftrightarrow \limsup _{n \rightarrow \infty}\left|\frac{g(n)}{f(n)}\right|=\infty .
\end{aligned}
$$

3. We use the notation $C^{0, \beta}(\mathcal{I})=\{f: \mathcal{I} \rightarrow \mathbb{C}:$ there is some $K>0$ such that $|f(x)-f(y)| \leq$ $\left.K|x-y|^{\beta}\right\}$ for $\beta \in(0,1]$.

\section{Trotter product formula and evolution semigroups}

Below we consider the Banach space $L^{p}(\mathcal{I}, X)$ for $\mathcal{I}:=[0, T], p \in[1, \infty)$. Recall that semigroup $\{\mathcal{U}(\tau)\}_{\tau \geq 0}$, on the Banach space $L^{p}(\mathcal{I}, X)$ is called an evolution semigroup if there is a propagator $\{U(t, s)\}_{(t, s) \in \Delta}$ such that the representation (1.5) holds.

Let $\mathcal{K}_{0}$ be the generator of an evolution semigroup $\left\{\mathcal{U}_{0}(\tau)\right\}_{\tau \geq 0}$ and let $\mathcal{B}$ be a multiplication operator induced by a measurable family $\{B(t)\}_{t \in \mathcal{I}}$ of generators of contraction semigroups. Note that in this case the multiplication operator $\mathcal{B}(1.8)$ is a generator of a contraction semigroup $\left(e^{-\tau \mathcal{B}} f\right)(t)=e^{-\tau B(t)} f(t)$, on the Banach space $L^{p}(\mathcal{I}, X)$. Since $\left\{\mathcal{U}_{0}(\tau)\right\}_{\tau \geq 0}$ is an evolution semigroup, then by definition (1.5) there is a propagator $\left\{U_{0}(t, s)\right\}_{(t, s) \in \Delta}$ such that the representation

$$
\left(\mathcal{U}_{0}(\tau) f\right)(t)=U_{0}(t, t-\tau) \chi_{\mathcal{I}}(t-\tau) f(t-\tau), \quad f \in L^{p}(\mathcal{I}, X),
$$

is valid for a.e. $t \in \mathcal{I}$ and $\tau \geq 0$. Then we define

$$
G_{j}(t, s ; n):=U_{0}\left(s+j \frac{(t-s)}{n}, s+(j-1) \frac{(t-s)}{n}\right) e^{-\frac{(t-s)}{n} B\left(s+(j-1) \frac{(t-s)}{n}\right)}
$$

where $j \in\{1,2, \ldots, n\}, n \in \mathbb{N},(t, s) \in \Delta$, and we set

$$
V_{n}(t, s):=\prod_{j=1}^{n \leftarrow} G_{j}(t, s ; n), \quad n \in \mathbb{N}, \quad(t, s) \in \Delta,
$$


where the product is increasingly ordered in $j$ from the right to the left. Then a straightforward computation shows that the representation

$$
\left(\left(e^{-\tau \mathcal{K}_{0} / n} e^{-\tau \mathcal{B} / n}\right)^{n} f\right)(t)=V_{n}(t, t-\tau) \chi_{\mathcal{I}}(t-\tau) f(t-\tau)
$$

$f \in L^{p}(\mathcal{I}, X)$, holds for each $\tau \geq 0$ and a.e. $t \in \mathcal{I}$.

Proposition 2.1. Let $\mathcal{K}$ and $\mathcal{K}_{0}$ be generators of evolution semigroups on the Banach space $L^{p}(\mathcal{I}, X)$ for some $p \in[1, \infty)$. Further, let $\{B(t) \in \mathcal{G}(1,0)\}_{t \in \mathcal{I}}$ be a strongly measurable family of generators of contraction on $X$ semigroups. Then

$$
\sup _{\tau \geq 0}\left\|e^{-\tau \mathcal{K}}-\left(e^{-\tau \mathcal{K}_{0} / n} e^{-\tau \mathcal{B} / n}\right)^{n}\right\|_{\mathcal{B}\left(L^{p}(\mathcal{I}, X)\right)}=\underset{(t, s) \in \Delta}{\operatorname{ess} \sup }\left\|U(t, s)-V_{n}(t, s)\right\|_{\mathcal{B}(X)}, \quad n \in \mathbb{N} .
$$

Proof. Let $\{L(\tau)\}_{\tau \geq 0}$ be the left-shift semigroup on the Banach space $\mathfrak{X}=L^{p}(\mathcal{I}, X)$ :

$$
(L(\tau) f)(t)=\chi_{\mathcal{I}}(t+\tau) f(t+\tau), \quad f \in L^{p}(\mathcal{I}, X) .
$$

Using that we get

$$
\left(L(\tau)\left(e^{-\tau \mathcal{K}}-\left(e^{-\tau / n \mathcal{K}_{0}} e^{-\tau \mathcal{B} / n}\right)^{n}\right) f\right)(t)=\left\{U(t+\tau, t)-V_{n}(t+\tau, t)\right\} \chi_{\mathcal{I}}(t+\tau) f(t)
$$

for $\tau \geq 0$ and a.e. $t \in \mathcal{I}$. It turns out that for each $n \in \mathbb{N}$ the operator $L(\tau)\left(e^{-\tau \mathcal{K}}-\left(e^{-\tau / n \mathcal{K}_{0}} e^{-\tau \mathcal{B} / n}\right)^{n}\right)$ is a multiplication operator induced by $\left\{\left(U(t+\tau, t)-V_{n}(t+\tau, t)\right) \chi_{\mathcal{I}}(t+\tau)\right\}_{t \in \mathcal{I}}$. Therefore,

$$
\left\|L(\tau)\left(e^{-\tau \mathcal{K}}-\left(e^{-\tau \mathcal{K}_{0} / n} e^{-\tau \mathcal{B} / n}\right)^{n}\right)\right\|_{\mathcal{B}(\mathfrak{X})}=\operatorname{ess} \sup _{t \in \mathcal{I}}\left\|U(t+\tau, t)-V_{n}(t+\tau, t)\right\|_{\mathcal{B}(X)} \chi_{\mathcal{I}}(t+\tau),
$$

for each $\tau \geq 0$. Note that one has

$$
\sup _{\tau \geq 0}\left\|L(\tau)\left(e^{-\tau \mathcal{K}}-\left(e^{-\tau \mathcal{K}_{0} / n} e^{-\tau \mathcal{B} / n}\right)^{n}\right)\right\|_{\mathcal{B}(\mathfrak{X})}=\underset{\tau \geq 0}{\operatorname{ess} \sup }\left\|L(\tau)\left(e^{-\tau \mathcal{K}}-\left(e^{-\tau \mathcal{K}_{0} / n} e^{-\tau \mathcal{B} / n}\right)^{n}\right)\right\|_{\mathcal{B}(\mathfrak{X})} .
$$

This is based on the fact that if $F(\cdot): \mathbb{R}_{+} \longrightarrow \mathcal{B}(\mathfrak{X})$ is strongly continuous, then $\sup _{\tau \geq 0}\|F(\tau)\|_{\mathcal{B}(\mathfrak{X})}=$ $\operatorname{ess} \sup _{\tau \geq 0}\|F(\tau)\|_{\mathcal{B}(\mathfrak{X})}$. Hence, we find

$$
\left.\sup _{\tau \geq 0}\left\|L(\tau)\left(e^{-\tau \mathcal{K}}-\left(e^{-\tau \mathcal{K}_{0} / n} e^{-\tau \mathcal{B} / n}\right)^{n}\right)\right\|_{\mathcal{B}(\mathfrak{X})}=\underset{\tau \geq 0}{\operatorname{ess} \sup } \operatorname{ess} \sup _{t \in \mathcal{I}} \| U(t+\tau, t)-V_{n}(t+\tau, t)\right) \|_{\mathcal{B}(X)} \chi_{\mathcal{I}}(t+\tau) .
$$

Further, if $\Phi(\cdot, \cdot): \mathbb{R}_{+} \times \mathcal{I} \longrightarrow \mathcal{B}(X)$ is a strongly measurable function, then

$$
\underset{(\tau, t) \in \mathbb{R}_{+} \times \mathcal{I}}{\operatorname{ess} \sup }\|\Phi(\tau, t)\|_{\mathcal{B}(X)}=\underset{\tau \geq 0}{\operatorname{ess} \sup } \underset{t \in \mathcal{I}}{\operatorname{ess} \sup }\|\Phi(\tau, t)\|_{\mathcal{B}(X)} .
$$

Then, taking into account two last equalities, one obtains

$$
\begin{aligned}
\sup _{\tau \geq 0}\left\|L(\tau)\left(e^{-\tau \mathcal{K}}-\left(e^{-\tau \mathcal{K}_{0} / n} e^{-\tau \mathcal{B} / n}\right)^{n}\right)\right\|_{\mathcal{B}(\mathfrak{X})} & =\operatorname{ess~sup}_{(\tau, t) \in \mathbb{R}_{+} \times \mathcal{I}}\left\|U(t+\tau, t)-V_{n}(t+\tau, t)\right\|_{\mathcal{B}(X)} \chi_{\mathcal{I}}(t+\tau)= \\
& =\underset{(t, s) \in \Delta}{\operatorname{ess} \sup }\left\|U(t, s)-V_{n}(t, s)\right\|_{\mathcal{B}(X)},
\end{aligned}
$$

that proves (2.2) 


\section{Bounded perturbations of the shift semigroup generator}

\subsection{Basic facts}

We study bounded perturbations of the evolution generator $D_{0}$ (1.6). To do this aim we consider $\mathcal{I}=[0,1]$, $X=\mathbb{C}$ and we denote by $L^{p}(\mathcal{I})$ the Banach space $L^{p}(\mathcal{I}, \mathbb{C})$.

For $t \in \mathcal{I}$, let $q: t \mapsto q(t) \in L^{\infty}(\mathcal{I})$. Then, $q$ induces a bounded multiplication operator $Q$ on the Banach space $L^{p}(\mathcal{I})$ :

$$
(Q f)(t)=q(t) f(t), \quad f \in L^{p}(\mathcal{I}) .
$$

For simplicity we assume that $q \geq 0$. Then $Q$ generates on $L^{p}(\mathcal{I})$ a contraction semigroup $\left\{e^{-\tau Q}\right\}_{\tau \geq 0}$. Since generator $Q$ is bounded, the closed operator $\mathcal{A}:=D_{0}+Q$, with $\operatorname{domain} \operatorname{dom}(\mathcal{A})=\operatorname{dom}\left(D_{0}\right)$, is generator of a semigroup on $L^{p}(\mathcal{I})$. By [7], the Trotter product formula in the strong topology follows immediately

$$
\left(e^{-\tau D_{0} / n} e^{-\tau Q / n}\right)^{n} f \rightarrow e^{-\tau\left(D_{0}+Q\right)} f, \quad f \in L^{p}(\mathcal{I})
$$

uniformly in $\tau \in[0, T]$ on bounded time intervals.

Following [2, §5], we define on $X=\mathbb{C}$ a family of bounded operators $\{V(t)\}_{t \in \mathcal{I}}$ by

$$
V(t):=e^{-\int_{0}^{t} d s q(s)}
$$

Note that for almost every $t \in \mathcal{I}$ these operators are positive. Then $V^{-1}(t)$ exists and it has the form

$$
V^{-1}(t)=e^{\int_{0}^{t} d s q(s)} .
$$

The operator families $\{V(t)\}_{t \in \mathcal{I}}$ and $\left\{V^{-1}(t)\right\}_{t \in \mathcal{I}}$ induce two bounded multiplication operators $\mathcal{V}$ and $\mathcal{V}^{-1}$ on $L^{p}(\mathcal{I})$, respectively. Then invertibility implies that $\mathcal{V} \mathcal{V}^{-1}=\mathcal{V}^{-1} \mathcal{V}=\left.I d\right|_{L^{p}}$. Using the operator $\mathcal{V}$ one easily verifies that $D_{0}+Q$ is similar to $D_{0}$, i.e. one has

$$
\mathcal{V}^{-1}\left(D_{0}+Q\right) \mathcal{V}=D_{0}, \quad \text { or } \quad D_{0}+Q=\mathcal{V} D_{0} \mathcal{V}^{-1}
$$

Hence, the semigroup generated on $L^{p}(\mathcal{I})$ by $D_{0}+Q$ gets the explicit form:

$$
\left(e^{-\tau\left(D_{0}+Q\right)} f\right)(t)=\left(\mathcal{V} e^{-\tau D_{0}} \mathcal{V}^{-1} f\right)(t)=e^{-\int_{t-\tau}^{t} q(y) d y} f(t-\tau) \chi_{\mathcal{I}}(t-\tau) .
$$

Since by (1.5) the propagator $U(t, s)$ that corresponds to evolution semigroup (3.2) is defined by

$$
\left(e^{-\tau\left(D_{0}+Q\right)}\right) f(t)=U(t, t-\tau) f(t-\tau) \chi_{\mathcal{I}}(t-\tau),
$$

we deduce that it is equal to $U(t, s)=e^{-\int_{s}^{t} d y q(y)}$.

Now we study the corresponding Trotter product formula. For a fixed $\tau \geq 0$ and $n \in \mathbb{N}$, we define approximation $V_{n}$ by

$$
\left(\left(e^{-\tau D_{0} / n} e^{-\tau Q / n}\right)^{n} f\right)(t)=: V_{n}(t, t-\tau) \chi_{\mathcal{I}}(t-\tau) f(t-\tau) .
$$

Then by straightforward calculations, similar to (2.1), one finds that

$$
V_{n}(t, s)=e^{-\frac{t-s}{n} \sum_{k=0}^{n-1} q\left(s+k \frac{t-s}{n}\right)}, \quad(t, s) \in \Delta .
$$


Proposition 3.1. Let $q \in L^{\infty}(\mathcal{I})$ be non-negative. Then

$$
\sup _{\tau \geq 0}\left\|e^{-\tau\left(D_{0}+Q\right)}-\left(e^{-\tau D_{0} / n} e^{-\tau Q / n}\right)^{n}\right\|_{\mathcal{B}\left(L^{p}(\mathcal{I})\right)}=\Theta\left(\operatorname{ess} \sup _{(t, s) \in \Delta}\left|\int_{s}^{t} q(y) d y-\frac{t-s}{n} \sum_{k=0}^{n-1} q\left(s+k \frac{t-s}{n}\right)\right|\right)
$$

as $n \rightarrow \infty$, where $\Theta$ is the Landau symbol defined in Section 1 .

Proof. First, by Proposition 2.1 and by $U(t, s)=e^{-\int_{s}^{t} d y q(y)}$ we obtain

$$
\sup _{\tau \geq 0}\left\|e^{-\tau\left(D_{0}+Q\right)}-\left(e^{-\tau D_{0} / n} e^{-\tau Q / n}\right)^{n}\right\|_{\mathcal{B}\left(L^{p}(\mathcal{I})\right)}=\underset{(t, s) \in \Delta}{\operatorname{ess} \sup }\left|e^{-\int_{s}^{t} d y q(y)}-e^{-\frac{t-s}{n} \sum_{k=0}^{n-1} q\left(s+k \frac{t-s}{n}\right)}\right| .
$$

Then, using the inequality

$$
e^{-\max \{x, y\}}|x-y| \leq\left|e^{-x}-e^{-y}\right| \leq|x-y|, \quad 0 \leq x, y,
$$

for $0 \leq s<t \leq 1$ one finds the estimates

$$
e^{-\|q\|_{L^{\infty}}} R_{n}(t, s ; q) \leq\left|e^{-\int_{s}^{t} d y q(y)}-e^{-\frac{t-s}{n} \sum_{k=0}^{n-1} q\left(s+k \frac{t-s}{n}\right)}\right| \leq R_{n}(t, s ; q),
$$

where

$$
R_{n}(t, s, q):=\left|\int_{s}^{t} d y q(y)-\frac{t-s}{n} \sum_{k=0}^{n-1} q\left(s+k \frac{t-s}{n}\right)\right|, \quad(t, s) \in \Delta .
$$

Hence, for the left-hand side of (3.4) we get the estimate

$$
e^{-\|q\|_{L^{\infty}}} R_{n}(q) \leq \sup _{\tau \geq 0}\left\|e^{-\tau\left(D_{0}+Q\right)}-\left(e^{-\tau D_{0} / n} e^{-\tau Q / n}\right)^{n}\right\|_{\mathcal{B}\left(L^{p}\right)} \leq R_{n}(q)
$$

where $R_{n}(q):=\operatorname{ess}_{\sup }(t, s) \in \Delta R_{n}(t, s ; q), n \in \mathbb{N}$. These estimates together with definition of $\Theta$ prove the assertion.

Note that by virtue of (3.5) and Proposition 3.1 the operator-norm convergence rate of the Trotter product formula for the pair $\left\{D_{0}, Q\right\}$ coincides with the convergence rate of the integral Darboux-Riemann sum approximation of the Lebesgue integral.

\subsection{Examples}

First we consider the case of a real Hölder-continuous function $q \in C^{0, \beta}(\mathcal{I})$.

Theorem 3.2. If $q \in C^{0, \beta}(\mathcal{I})$ is non-negative, then

$$
\sup _{\tau \geq 0}\left\|e^{-\tau\left(D_{0}+Q\right)}-\left(e^{-\tau D_{0} / n} e^{-\tau Q / n}\right)^{n}\right\|=O\left(1 / n^{\beta}\right)
$$

as $n \rightarrow \infty$.

Proof. One has

$$
\int_{s}^{t} d y q(y)-\frac{t-s}{n} \sum_{k}^{n-1} q\left(s+\frac{k}{n}(t-s)\right)=\sum_{k=0}^{n-1} \int_{\frac{k}{n}(t-s)}^{\frac{k+1}{n}(t-s)} d y\left(q(s+y)-q\left(s+\frac{k}{n}(t-s)\right)\right)
$$


which yields the estimate

$$
\left|\int_{s}^{t} d y q(y)-\frac{t-s}{n} \sum_{k}^{n-1} q\left(s+\frac{k}{n}(t-s)\right)\right| \leq \sum_{k=0}^{n-1} \int_{\frac{k}{n}(t-s)}^{\frac{k+1}{n}(t-s)} d y\left|q(s+y)-q\left(s+\frac{k}{n}(t-s)\right)\right| .
$$

Since $q \in C^{0, \beta}(\mathcal{I})$, there is a constant $L_{\beta}>0$ such that for $y \in\left[\frac{k}{n}(t-s), \frac{k+1}{n}(t-s)\right]$ one has

$$
\mid q(s+y)-q\left(s+\frac{k}{n}(t-s)\left|\leq L_{\beta}\right| y-\left.\frac{k}{n}(t-s)\right|^{\beta} \leq L_{\beta} \frac{(t-s)^{\beta}}{n^{\beta}} .\right.
$$

Hence, we find

$$
\left|\int_{s}^{t} q(y) d y-\frac{t-s}{n} \sum_{k}^{n-1} q\left(s+\frac{k}{n}(t-s)\right)\right| \leq L_{\beta} \frac{(t-s)^{1+\beta}}{n^{\beta}} \leq L_{\beta} \frac{1}{n^{\beta}},
$$

which proves

$$
\underset{(t, s) \in \Delta}{\operatorname{ess} \sup }\left|\int_{s}^{t} q(y) d y-\frac{t-s}{n} \sum_{k}^{n-1} q\left(s+\frac{k}{n}(t-s)\right)\right|=O\left(\frac{1}{n^{\beta}}\right) .
$$

Applying now Proposition 3.1 one completes the proof.

It is a natural question: what happens, when $q$ is only continuous?

Theorem 3.3. If $q: \mathcal{I} \rightarrow \mathbb{C}$ is continuous and non-negative, then

$$
\left\|e^{-\tau\left(D_{0}+Q\right)}-\left(e^{-\tau D_{0} / n} e^{-\tau Q / n}\right)^{n}\right\|=o(1),
$$

as $n \rightarrow \infty$.

Proof. Since $q(\cdot)$ is continuous, then for any $\varepsilon>0$ there is $\delta>0$ such that for $|y-x|<\delta$ we have $|q(y)-q(x)|<\varepsilon, y, x \in \mathcal{I}$. Therefore, if $1 / n<\delta$, then for $y \in\left(\frac{k}{n}(t-s), \frac{k+1}{n}(t-s)\right)$ we have

$$
\left|q(s+y)-q\left(s+\frac{k}{n}(t-s)\right)\right|<\varepsilon, \quad(t, s) \in \Delta .
$$

Hence,

$$
\left|\int_{s}^{t} q(y) d y-\frac{t-s}{n} \sum_{k}^{n-1} q\left(s+\frac{k}{n}(t-s)\right)\right| \leq \varepsilon(t-s) \leq \varepsilon
$$

which yields

$$
\underset{(t, s) \in \Delta}{\operatorname{ess} \sup }\left|\int_{s}^{t} q(y) d y-\frac{t-s}{n} \sum_{k}^{n-1} q\left(s+\frac{k}{n}(t-s)\right)\right|=o(1) .
$$

Now it remains only to apply Proposition 3.1

We comment that for a general continuous $q$ one can say nothing about the convergence rate. Indeed, it can be shown that in (3.6) the convergence to zero can be arbitrary slow.

Theorem 3.4. Let $\delta_{n}>0$ be a sequence with $\delta_{n} \rightarrow 0$ as $n \rightarrow \infty$. Then there exists a continuous function $q: \mathcal{I}=[0,1] \rightarrow \mathbb{R}$ such that

$$
\sup _{\tau \geq 0}\left\|e^{-\tau\left(D_{0}+Q\right)}-\left(e^{-\tau D_{0} / n} e^{-\tau Q / n}\right)^{n}\right\|_{\mathcal{B}\left(L^{p}(\mathcal{I})\right)}=\omega\left(\delta_{n}\right)
$$

as $n \rightarrow \infty$, where $\omega$ is the Landau symbol defined in Section 1 . 
Proof. Taking into account Theorem 6 of [8], we find that for any sequence $\left\{\delta_{n}\right\}_{n \in \mathbb{N}}, \delta_{n}>0$ satisfying $\lim _{n \rightarrow \infty} \delta_{n}=0$ there exists a continuous function $f(\cdot):[0,2 \pi] \longrightarrow \mathbb{R}$ such that

$$
\left|\int_{0}^{2 \pi} f(x) d x-\frac{2 \pi}{n} \sum_{k=1}^{n} f(2 k \pi / n)\right|=\omega\left(\delta_{n}\right)
$$

as $n \rightarrow \infty$. Setting $q(y):=f(2 \pi(1-y)), y \in[0,1]$, we get a continuous function $q(\cdot):[0,1] \longrightarrow \mathbb{R}$, such that

$$
\left|\int_{0}^{1} q(y) d y-\frac{1}{n} \sum_{k=0}^{n-1} q(k / n)\right|=\omega\left(\delta_{n}\right) .
$$

Because $q(\cdot)$ is continuous we find

$$
\underset{(t, s) \in \Delta}{\operatorname{ess} \sup }\left|\int_{s}^{t} q(y) d y-\frac{t-s}{n} \sum_{n=0}^{n-1} q\left(s+k \frac{t-s}{n}\right)\right| \geq\left|\int_{0}^{1} q(y) d y-\frac{1}{n} \sum_{k=0}^{n-1} q(k / n)\right|,
$$

which yields

$$
\underset{(t, s) \in \Delta}{\operatorname{ess~sup}}\left|\int_{s}^{t} q(y) d y-\frac{t-s}{n} \sum_{n=0}^{n-1} q\left(s+k \frac{t-s}{n}\right)\right|=\omega\left(\delta_{n}\right) .
$$

Applying now Proposition 3.1 we prove (3.7).

Our final comment concerns the case when $q$ is only measurable. Then it can happen that the Trotter product formula for that pair $\left\{D_{0}, Q\right\}$ does not converge in the operator-norm topology.

Theorem 3.5. There is a non-negative function $q \in L^{\infty}([0,1])$ such that

$$
\limsup _{n \rightarrow \infty} \sup _{\tau \geq 0}\left\|e^{-\tau\left(D_{0}+Q\right)}-\left(e^{-\tau D_{0} / n} e^{-\tau Q / n}\right)^{n}\right\|_{\mathcal{B}\left(L^{p}(\mathcal{I})\right)}>0
$$

Proof. Let us introduce the open intervals

$$
\begin{aligned}
\Delta_{0, n} & :=\left(0, \frac{1}{2^{2 n+2}}\right), \\
\Delta_{k, n} & :=\left(t_{k, n}-\frac{1}{2^{2 n+2}}, t_{k, n}+\frac{1}{2^{2 n+2}}\right), \quad k=1,2, \ldots, 2^{n}-1, \\
\Delta_{2^{n}, n} & :=\left(1-\frac{1}{2^{2 n+2}}, 1\right),
\end{aligned}
$$

$n \in \mathbb{N}$, where

$$
t_{k, n}=\frac{k}{2^{n}}, \quad k=0, \ldots, n, \quad n \in \mathbb{N} .
$$

Notice that $t_{0, n}=0$ and $t_{2^{n}, n}=1$. One easily checks that the intervals $\Delta_{k, n}, k=0, \ldots, 2^{n}$, are mutually disjoint. We introduce the open sets

$$
\mathcal{O}_{n}=\bigcup_{k=0}^{2^{n}} \Delta_{k, n} \subseteq \mathcal{I}, \quad n \in \mathbb{N}
$$

and

$$
\mathcal{O}=\bigcup_{n \in \mathbb{N}} \mathcal{O}_{n} \subseteq \mathcal{I}
$$

Then it is clear that

$$
\left|\mathcal{O}_{n}\right|=\frac{1}{2^{n+1}}, \quad n \in \mathbb{N}, \quad \text { and } \quad|\mathcal{O}| \leq \frac{1}{2}
$$


Therefore, the Lebesgue measure of the closed set $\mathcal{C}:=\mathcal{I} \backslash \mathcal{O} \subseteq \mathcal{I}$ can be estimated by

$$
|\mathcal{C}| \geq \frac{1}{2}
$$

Using the characteristic function $\chi_{\mathcal{C}}(\cdot)$ of the set $\mathcal{C}$ we define

$$
q(t):=\chi_{\mathcal{C}}(t), \quad t \in \mathcal{I} .
$$

The function $q(\cdot)$ is measurable and it satisfies $0 \leq q(t) \leq 1, t \in \mathcal{I}$.

Let $\varepsilon \in(0,1)$. We choose $s \in(0, \varepsilon)$ and $t \in(1-\varepsilon, 1)$ and we set

$$
\xi_{k, n}(t, s):=s+k \frac{t-s}{2^{n}}, \quad k=0, \ldots, 2^{n}-1, \quad n \in \mathbb{N}, \quad(t, s) \in \Delta .
$$

Note that $\xi_{k, n}(t, s) \in(0,1), k=0, \ldots, 2^{n}-1, n \in \mathbb{N}$. Moreover, we have

$$
t_{k, n}-\xi_{k, n}(t, s)=k \frac{1}{2^{n}}-s-k \frac{t-s}{2^{n}}=k \frac{1-t+s}{2^{n}}-s
$$

which leads to the estimate

$$
\left|t_{k, n}-\xi_{k, n}(t, s)\right| \leq \varepsilon\left(\frac{k}{2^{n-1}}+1\right), \quad k=0, \ldots, 2^{n}-1, \quad n \in \mathbb{N}
$$

Hence

$$
\left|t_{k, n}-\xi_{k, n}(t, s)\right| \leq 3 \varepsilon, \quad k=0, \ldots, 2^{n}-1, \quad n \in \mathbb{N}
$$

Let $\varepsilon_{n}:=1 /\left(3 \cdot 2^{2 n+2}\right)$ for $n \in \mathbb{N}$. Then we get that $\xi_{k, n}(t, s) \in \Delta_{k, n}$ for $k=0, \ldots, 2^{n}-1, n \in \mathbb{N}$, $s \in\left(0, \varepsilon_{n}\right)$ and for $t \in\left(1-\varepsilon_{n}, 1\right)$.

Now let

$$
S_{n}(t, s ; q):=\frac{t-s}{n} \sum_{k=0}^{n-1} q\left(s+k \frac{t-s}{n}\right), \quad n \in \mathbb{N}, \quad(t, s) \in \Delta .
$$

We consider

$$
S_{2^{n}}(t, s ; q)=\frac{t-s}{n} \sum_{k=0}^{2^{n}-1} q\left(s+k \frac{t-s}{2^{n}}\right)=\frac{t-s}{n} \sum_{k=0}^{2^{n}-1} q\left(\xi_{k, n}(t, s)\right),
$$

$n \in \mathbb{N},(t, s) \in \Delta$. If $s \in\left(0, \varepsilon_{n}\right)$ and $t \in\left(1-\varepsilon_{n}, 1\right)$, then $S_{2^{n}}(t, s ; q)=0, n \in \mathbb{N}$ and

$$
\left|\int_{s}^{t} q(y) d y-S_{2^{n}}(t, s ; q)\right|=\int_{s}^{t} q(y) d y, \quad n \in \mathbb{N},
$$

for $s \in\left(0, \varepsilon_{n}\right)$ and $t \in\left(1-\varepsilon_{n}, 1\right)$. In particular, this yields

$$
\underset{(t, s) \in \Delta}{\operatorname{ess} \sup _{\Delta}}\left|\int_{s}^{t} q(y) d y-S_{2^{n}}(t, s ; q)\right| \geq \underset{(t, s) \in \Delta}{\operatorname{ess} \sup _{s}} \int_{s}^{t} q(y) d y \geq \int_{\mathcal{I}} \chi_{\mathcal{C}}(y) d y \geq \frac{1}{2} .
$$

Hence, we obtain

$$
\limsup _{n \rightarrow \infty} \operatorname{ess~sup}_{(t, s) \in \Delta}\left|\int_{s}^{t} q(y) d y-S_{2^{n}}(t, s ; q)\right| \geq \frac{1}{2},
$$

and applying Proposition 3.1 we finish the prove of (3.8).

We note that Theorem 3.5 does not exclude the convergence of the Trotter product formula for the pair $\left\{D_{0}, Q\right\}$ in the strong operator topology. Examples of this dichotomy are known for the Trotter-Kato product formula in Hilbert spaces [3]. By virtue of (3.1) and (3.8), Theorem 3.5 yields an example of this dichotomy in Banach spaces. 


\section{Acknowledgments}

The preparation of the paper was supported by the European Research Council via ERC-2010-AdG no 267802 ("Analysis of Multiscale Systems Driven by Functionals"). V.A.Z. thanks WIAS for hospitality.

\section{References}

[1] Vincent Cachia and Valentin A. Zagrebnov. Operator-norm convergence of the Trotter product formula for holomorphic semigroups. J. Oper. Theory, 46(1):199-213, 2001.

[2] Paul R.Chernoff. Product formulas, nonlinear semigroups, and addition of unbounded operators. Memoirs of the American Mathematical Society, No. 140. American Mathematical Society, Providence, R. I., 1974.

[3] Takashi Ichinose, Hideo Tamura, Hiroshi Tamura, and Valentin A. Zagrebnov. Note on the paper: "The norm convergence of the Trotter-Kato product formula with error bound" by T. Ichinose and H. Tamura. Comm. Math. Phys., 221(3):499-510, 2001.

[4] Tosio Kato. Trotter's product formula for an arbitrary pair of self-adjoint contraction semigroups. Topics in functional analysis, Essays dedic. M. G. Krein, Adv. Math., Suppl. Stud. 3, 185-195, 1978.

[5] Tosio Kato. Perturbation theory for linear operators. Classics in Mathematics. Springer-Verlag, Berlin, 1995.

[6] Hagen Neidhardt, Artur Stephan, and Valentin A. Zagrebnov. Convergence rate estimates for the Trotter product approximations of solution operators for non-autonomous Cauchy problems. arXiv:1612.06147v1 [math.FA], December 2016.

[7] Hale F. Trotter. On the product of semi-groups of operators. Proc. Amer. Math. Soc., 10:545-551, 1959.

[8] J. L. Walsh and W. E. Sewell. Note on degree of approximation to an integral by riemann sums. The American Mathematical Monthly, 44(3):155-160, 1937. 